\title{
The role of toxin A and toxin B in Clostridium difficile infection
}

Sarah A. Kuehne*, Stephen T. Cartman*, John T. Heap, Michelle L. Kelly, Alan

Cockayne \& Nigel P. Minton

Centre for Biomolecular Sciences, School of Molecular Medical Sciences, Nottingham

Digestive Diseases Centre, NIHR Biomedical Research Unit, University of Nottingham, Nottingham, NG7 2RD, UK

*These authors contributed equally to this work.

Clostridium difficile infection is the leading cause of healthcare associated diarrhoea in Europe and North America ${ }^{1,2}$. During infection, $C$. difficile produces two key virulence determinants, toxin A and toxin B. Experiments with purified toxins have suggested that toxin $A$ alone is able to evoke the symptoms of $C$. difficile infection, but toxin $B$ is unable to do so unless it is mixed with toxin A, or there is prior damage to the gut mucosa ${ }^{3}$. However, a recent study suggested that toxin $B$ is essential for $C$. difficile virulence and that a strain producing toxin $A$ alone was avirulent ${ }^{4}$. This creates a paradox over the individual importance of toxin A and toxin B. Here we show that isogenic mutants of $C$. difficile producing either toxin A or toxin B alone can cause fulminant disease in the hamster model of infection. By using a gene knock-out system ${ }^{5,6}$ to permanently inactivate the toxin genes, we found that $C$. difficile producing either one or both toxins displayed cytotoxic activity in vitro, which translated directly into virulence in vivo. Furthermore, by constructing the first ever double mutant strain of $C$. difficile, in which both toxin genes were inactivated, we were able to completely attenuate virulence. Our findings re-establish the importance of both toxin A and toxin B and highlight the need to continue considering both toxins in the development of diagnostic tests and effective counter-measures against $C$. difficile. 
Toxin A and toxin B both catalyse the glucosylation, and hence inactivation, of Rho-GTPases; small regulatory proteins of the eukaryotic actin cell cytoskeleton. This leads to disorganisation of the cell cytoskeleton and cell death ${ }^{7}$. The toxin genes, $t c d A$ and $t c d B$, are situated on the $C$. difficile chromosome in a 19.6 kilobase pathogenicity locus (PaLoc), along with the three accessory genes, $t c d C$, $t c d R$ and $t c d E$ (Fig. 1a). To address the individual importance of toxin A and toxin B, we used the ClosTron gene knock-out system ${ }^{6}$ to inactivate the toxin genes of $C$. difficile. This system inactivates genes by inserting an intron into the protein-encoding DNA sequence of a gene, thus resulting in a truncated and non-functional protein. The intron sequence itself encompasses an erythromycin resistance determinant which permits selective isolation of mutants. Furthermore, it has been shown experimentally that the insertions are completely stable, meaning that inactivation of a gene is permanent ${ }^{5}$.

Using the ClosTron system, we targeted insertions to $t c d A$ and $t c d B$ at nucleotide positions 1584 and 1511, respectively (Fig. 1a). In both cases, this placed the intron within DNA sequence encoding the toxin catalytic domain. Three separate isogenic mutants of the toxin A-positive, toxin B-positive $\left(\mathrm{A}^{+} \mathrm{B}^{+}\right)$C. difficile strain $630 \Delta \mathrm{erm}^{8}$ were constructed; two 'single-mutants', with toxin profiles $\mathrm{A}^{-} \mathrm{B}^{+}$and $\mathrm{A}^{+} \mathrm{B}^{-}$, respectively, and a 'double-mutant' with toxin profile $\mathrm{A}^{-} \mathrm{B}^{-}$. The $\mathrm{A}^{-} \mathrm{B}^{-}$double-mutant was made from the $\mathrm{A}^{+} \mathrm{B}^{-}$single-mutant by targeting $t c d A$ with a second intron which carried the chloramphenicol / thiamphenicol resistance gene catP instead of the usual erythromycin resistance determinant.

The genotype of each toxin mutant was characterised by PCR and DNA sequence analysis to confirm the exact location of each intron insertion made (data not shown). Southern blot analysis of EcoRV-digested genomic DNA samples, using an intron- 
specific probe, confirmed that the $\mathrm{A}^{-} \mathrm{B}^{+}$and $\mathrm{A}^{+} \mathrm{B}^{-}$mutants each had a single insertion, while the $\mathrm{A}^{-} \mathrm{B}^{-}$mutant had a double insertion (Fig. 1b). It is noteworthy that three bands were expected for the $\mathrm{A}^{-} \mathrm{B}^{-}$double-mutant strain because the catP gene harbours an EcoRV site. The phenotype of each strain was confirmed by Western blot analysis. Use of a toxin A-specific antibody-probe confirmed that the $\mathrm{AB}^{-}$and $\mathrm{A}^{-} \mathrm{B}^{-}$mutants no longer produced toxin A (Fig. 1c). Likewise, use of a toxin B-specific antibody-probe confirmed that the $\mathrm{A}^{+} \mathrm{B}^{-}$and $\mathrm{A}^{-} \mathrm{B}^{-}$mutants no longer produced toxin $\mathrm{B}$ (Fig. 1d).

Subsequently, in vitro cell cytotoxicity assays were carried out using HT29 (human colon carcinoma) cells and Vero (African green monkey kidney) cells. Each of these cell lines is susceptible to both toxin A and toxin B, although HT29 cells are more sensitive to toxin $\mathrm{A}$ and Vero cells are more sensitive to toxin $\mathrm{B}^{9}$. The action of toxin $\mathrm{A}$ and toxin B causes the cells to 'round' (that is, lose morphology) and die; a phenomenon which is clearly visible by light microscopy. We incubated cultured cells for $24 \mathrm{~h}$ with 4-fold dilution series of $C$. difficile culture supernatants. To obtain the most objective data set possible, we determined the endpoint titre of each dilution series, rather than implementing a subjective cell scoring system. Endpoint titre was defined as the first dilution in a series for which HT29 or Vero cell morphology was indistinguishable from the negative controls (that is, cells which had been incubated with uninoculated $C$. difficile culture medium).

As expected, the $\mathrm{A}^{-} \mathrm{B}^{-}$double toxin mutant did not display any cytotoxic activity towards either HT29 or Vero cells (Fig. 2a,b). Compared to the $\mathrm{A}^{+} \mathrm{B}^{+}$parental strain, the $\mathrm{A}^{-}{ }^{+}$mutant displayed reduced toxicity towards HT29 cells, although the difference was not statistically significant, and a similar degree of toxicity towards Vero cells (Fig 2a,b). These findings were anticipated given the respective sensitivities of HT29 cells and Vero cells to toxin $\mathrm{A}$ and toxin $\mathrm{B}^{9}$. However, unexpectedly, when compared to the $\mathrm{A}^{+} \mathrm{B}^{+}$parental strain, the $\mathrm{A}^{+} \mathrm{B}^{-}$mutant displayed increased toxicity towards HT29 cells, 
although the difference was not statistically significant, and a similar degree of toxicity towards Vero cells (Fig. 2a,b). We reasoned that this may occur due to increased expression of toxin $\mathrm{A}$ by the $\mathrm{A}^{+} \mathrm{B}^{-}$mutant; a phenomenon which has been reported previously ${ }^{4}$. Indeed, qRT-PCR analysis confirmed that expression of toxin A was an average of 3.3-fold greater in the $\mathrm{A}^{+} \mathrm{B}^{-}$mutant than the $\mathrm{A}^{+} \mathrm{B}^{+}$parental strain (data not shown). We do not know the reason for this. However, considering the respective sensitivities of HT29 and Vero cells to toxin $\mathrm{A}^{9}$, this finding explains our unexpected cytotoxicity results, even accounting for the fact that the $\mathrm{A}^{+} \mathrm{B}^{-}$mutant does not produce any toxin B.

To confirm that the cytotoxic activity we had observed on HT29 cells and Vero cells were indeed attributable to the respective actions of toxin A and toxin $\mathrm{B}$, we carried out toxin neutralisation assays. Culture supernatants of each $C$. difficile toxin mutant and the $\mathrm{A}^{+} \mathrm{B}^{+}$parental strain were incubated with either toxin $\mathrm{A}$-specific or toxin B-specific neutralising antibodies, prior to inoculation onto HT29 and Vero cell monolayers. Importantly, culture supernatants were diluted equivalently such that, for the $\mathrm{A}^{+} \mathrm{B}^{+}$parental strain, only toxin A activity was detected on HT29 cells and only toxin B activity was detected on Vero cells (that is, toxin B activity towards HT29 cells was diluted-out completely and toxin A activity towards Vero cells was diluted-out completely) (Fig. 2c,d). As expected, the toxin A-specific antibody neutralised all toxic activity produced by the $\mathrm{A}^{+} \mathrm{B}^{-}$mutant and the toxin $\mathrm{B}$-specific antibody neutralized all toxic activity produced by the $\mathrm{A}^{-} \mathrm{B}^{+}$mutant (Fig. 2c,d). Interestingly, the increased production of toxin $\mathrm{A}$ by the $\mathrm{A}^{+} \mathrm{B}^{-}$mutant was clearly visible in this assay, as the cytotoxic activity of this strain towards Vero cells was not diluted-out completely as it was for the $\mathrm{A}^{+} \mathrm{B}^{+}$parental strain (Fig. 2d).

Having fully characterised our $C$. difficile toxin mutants in vitro, we tested the virulence of each in the hamster model of infection. Hamsters were each challenged 
with 100 spores of a single $C$. difficile strain, 5 days after an oral dose of clindamycin $(30 \mathrm{mg} / \mathrm{kg})$. Each toxin mutant and the $\mathrm{A}^{+} \mathrm{B}^{+}$parental strain were administered to eight hamsters in total. All hamsters became colonised by the $C$. difficile strain administered between 1 and 3 days post-challenge, with the exception of one which received the $\mathrm{A}^{+} \mathrm{B}^{-}$ single toxin mutant (Fig. 3a). Following colonisation, hamsters which received the $\mathrm{A}^{+} \mathrm{B}^{+}$ parental strain, the $\mathrm{A}^{-} \mathrm{B}^{+}$mutant or the $\mathrm{A}^{+} \mathrm{B}^{-}$mutant all developed symptoms of $C$. difficile infection, which resulted in a mean time to death of 1.0 day, 1.3 days and 4.0 days, respectively (Fig. 3b). In contrast, none of the hamsters colonised by the toxin null $\mathrm{A}^{-} \mathrm{B}^{-}$double mutant developed any symptoms of disease during the 14-day experimental period, indicating that this strain is completely attenuated for virulence. Bacteriological and PCR analysis of caecum samples taken from each hamster post mortem confirmed that the only infecting strain of $C$. difficile was, indeed, the strain administered in every case, thus ruling out any possibility of cross-contamination between cages or contamination from the environment (Supplementary Fig. 1).

In conclusion, it is clear that both toxin $\mathrm{A}$ and toxin $\mathrm{B}$ play an important role in $C$. difficile infection as we have shown here that a strain which produces either toxin on its own or both together is virulent. It is pertinent to question why we found that an $\mathrm{A}^{+} \mathrm{B}^{-}$ strain of $C$. difficile is virulent; a result which is in direct contrast with a similar study published recently ${ }^{4}$. This discrepancy may arise due to inherent differences between the hamsters used in each study. However, perhaps more likely is that there is one or more key differences between the strains of $C$. difficile studied. Although both strains are erythromycin-sensitive derivatives of strain $630^{10,11}$, they were isolated independently through serial sub-culture ${ }^{8,12}$. Therefore, either strain could have acquired one or more secondary mutations, which may affect the action of either one or both of the toxins. However, it is notable that our findings align with those of previous studies which have suggested a role for both toxin A and toxin B in C. difficile infection ${ }^{3,13-15}$. Moreover, given that the human colon is the principle site of pathology in patients infected with $C$. 
difficile, it stands to reason that an $\mathrm{A}^{+} \mathrm{B}^{-}$strain of $C$. difficile is virulent in vivo, as it is toxin A which displays the greatest cytotoxicity towards laboratory cultured human colon cells (that is, HT29 cells).

It is important to note that inherent variability exists between the toxins of some C. difficile strains; particularly in the case of toxin $\mathrm{B}^{16-19}$. In practical terms, this means that the toxins from different strains can vary in enzymatic activity (that is, different GTPase substrates may be glucosylated) and/or host-cell specificity. Consequently, it is not appropriate to over-interpret our findings and make general conclusions about the toxins produced by all toxigenic strains of $C$. difficile. Nonetheless, our results clearly demonstrate that a strain of $C$. difficile producing either toxin A or toxin B alone may be virulent and thus we have re-established the importance of both toxins in $C$. difficile infection.

It is interesting to note that a number of clinical cases of $C$. difficile infection have been attributed to naturally occurring $\mathrm{A}^{-} \mathrm{B}^{+}$strains ${ }^{20,21}$, but there have been no reports of naturally occurring $\mathrm{A}^{+} \mathrm{B}^{-}$isolates to date. This would suggest that $\mathrm{A}^{+} \mathrm{B}^{-}$strains do not exist, but it may also be an artefact of routine diagnostic testing practices. Either way, our results show that $\mathrm{A}^{+} \mathrm{B}^{-}$strains may be virulent and even if they do not exist in nature already, they may yet evolve. Consequently, it is imperative that both toxin A and toxin B continue to be considered in routine diagnostic settings and in the development of effective countermeasures against $C$. difficile.

\section{Methods Summary}

Mutants were constructed from the parental strain C. difficile $630 \Delta \mathrm{erm}^{8}$ using the ClosTron system ${ }^{5,6}$. The retargeted plasmids pMTL007C-E2::Cdi-tcdA-1584s, 
pMTL007C-E2::Cdi-tcdB-1511a and for the double pMTL007S-C7::Cdi-tcdA-1584s were transferred into $C$. difficile via conjugation. The single ClosTron mutants were isolated on erythromycin plates. The double mutant was isolated on thiamphenicol plates.

For cytotoxicity assays, the four strains were grown overnight in $5 \mathrm{ml} \mathrm{TY}$ under anaerobic conditions as previously described ${ }^{22}$. The cell densities were standardised before centrifugation and filtration. Supernatants were diluted in a 4-fold series and $20 \mu \mathrm{l}$ of dilutions were added onto monolayers of Vero and HT29 cells preincubated in 96 well plates for $48 \mathrm{~h}$ (at $37^{\circ} \mathrm{C}, 5 \% \mathrm{CO}_{2}$ ). Cytotoxicity was recorded after $24 \mathrm{~h}$. Statistical analysis was performed using one way ANOVA tests. For the neutralization assay appropriate dilutions of supernatants were pre-incubated with a suitable concentration of anti-TcdA or anti-TcdB serum (polyclonal, $\operatorname{tgcBIOMICS}$ ) for $1 \mathrm{~h}$ at $37^{\circ} \mathrm{C}$ and then added as previously described to Vero and HT29 cells and evaluated after $24 \mathrm{~h}$. Golden Syrian hamsters were dosed with clindamycin $(30 \mathrm{mg} / \mathrm{kg}) 5$ days prior to being infected orally with 100 spores each. Hamsters were monitored for signs of infection (including weight-loss, behavioural changes and wet-tail) and sacrificed when the endpoint was met. Faecal pellets were collected daily and plated to confirm the presence or absence of $C$. difficile. Caecum samples were homogenized, plated and C. difficile counts obtained. PCR was performed to determine the C. difficile genotype isolated from all samples. Supplementary Information, showing PCR results, is linked to the online version of the paper at www.nature.com/nature.

Full Methods and any associated references are available in the online version of the paper at www.nature.com/nature. 
1. Poutanen, S. M. \& Simor, A. E. Clostridium difficile-associated diarrhea in adults. Can Med Assoc J 171, 51-8 (2004).

2. Elliott, B., Chang, B. J., Golledge, C. L. \& Riley, T. V. Clostridium difficileassociated diarrhoea. Intern Med J 37, 561-8 (2007).

3. Lyerly, D. M., Saum, K. E., MacDonald, D. K. \& Wilkins, T. D. Effects of Clostridium difficile toxins given intragastrically to animals. Infect Immun 47, 349-52 (1985).

4. Lyras, D. et al. Toxin B is essential for virulence of Clostridium difficile. Nature 458, 1176-9 (2009).

5. Heap, J. T., Pennington, O. J., Cartman, S. T., Carter, G. P. \& Minton, N. $P$. The ClosTron: a universal gene knock-out system for the genus Clostridium. J Microbiol Methods 70, 452-64 (2007).

6. Heap, J. T. et al. The ClosTron: Mutagenesis in Clostridium refined and streamlined. J Microbiol Methods 80, 49-55 (2010).

7. Just, I. \& Gerhard, R. Large clostridial cytotoxins. Rev Physiol Biochem Pharmacol 152, 23-47 (2004).

8. Hussain, H. A., Roberts, A. P. \& Mullany, P. Generation of an erythromycin-sensitive derivative of Clostridium difficile strain 630 $(630 \Delta \mathrm{erm})$ and demonstration that the conjugative transposon $\mathrm{Tn} 916 \Delta \mathrm{E}$ enters the genome of this strain at multiple sites. J Med Microbiol 54, 137-41 (2005).

9. Torres, J., Camorlinga-Ponce, M. \& Munoz, O. Sensitivity in culture of epithelial cells from rhesus monkey kidney and human colon carcinoma to toxins A and B from Clostridium difficile. Toxicon 30, 419-26 (1992).

10. Sebaihia, M. et al. The multidrug-resistant human pathogen Clostridium difficile has a highly mobile, mosaic genome. Nat Genet 38, 779-86 (2006).

11. Hachler, H., Berger-Bachi, B. \& Kayser, F. H. Genetic characterization of a Clostridium difficile erythromycin-clindamycin resistance determinant that is transferable to Staphylococcus aureus. Antimicrob Agents Chemother 31, 1039-45 (1987).

12. O'Connor, J. R. et al. Construction and analysis of chromosomal Clostridium difficile mutants. Mol Microbiol 61, 1335-51 (2006).

13. Kim, P. H., laconis, J. P. \& Rolfe, R. D. Immunization of adult hamsters against Clostridium difficile-associated ileocecitis and transfer of protection to infant hamsters. Infect Immun 55, 2984-92 (1987).

14. Voth, D. E. \& Ballard, J. D. Clostridium difficile toxins: mechanism of action and role in disease. Clin Microbiol Rev 18, 247-63 (2005).

15. Du, T. \& Alfa, M. J. Translocation of Clostridium difficile toxin B across polarized Caco-2 cell monolayers is enhanced by toxin A. Can J Infect Dis 15, 83-8 (2004).

16. Chaves-Olarte, E. et al. R-Ras glucosylation and transient RhoA activation determine the cytopathic effect produced by toxin $B$ variants from toxin A-negative strains of Clostridium difficile. J Biol Chem 278, 7956-63 (2003).

17. Torres, J. F. Purification and characterisation of toxin B from a strain of Clostridium difficile that does not produce toxin A. J Med Microbiol 35, 40-4 (1991). 
18. Alfa, M. J. et al. Characterization of a toxin A-negative, toxin B-positive strain of Clostridium difficile responsible for a nosocomial outbreak of Clostridium difficile-associated diarrhea. J Clin Microbiol 38, 2706-14 (2000).

19. Stabler, R. A., Dawson, L. F., Phua, L. T. \& Wren, B. W. Comparative analysis of BI/NAP1/027 hypervirulent strains reveals novel toxin Bencoding gene (tcdB) sequences. J Med Microbiol 57, 771-5 (2008).

20. Drudy, D., Fanning, S. \& Kyne, L. Toxin A-negative, toxin B-positive Clostridium difficile. Int J Infect Dis 11, 5-10 (2007).

21. Drudy, D., Harnedy, N., Fanning, S., Hannan, M. \& Kyne, L. Emergence and control of fluoroquinolone-resistant, toxin A-negative, toxin B-positive Clostridium difficile. Infect Control Hosp Epidemiol 28, 932-40 (2007).

22. Heap, J. T., Pennington, O. J., Cartman, S. T. \& Minton, N. P. A modular system for Clostridium shuttle plasmids. J Microbiol Methods 78, 79-85 (2009).

Supplementary Information accompanies the paper on www.nature.com/nature.

Acknowledgements S.K., S.C., A.C. and N.M. acknowledge the financial support of the UK Medical Research Council, UK (G0601176). Support for M.K. was provided by the European Union (HEALTHF3-2008-223585) and for J.H. by the BBSRC (BB/F003390/1). We thank C. von-Eichel Streiber for supplying the neutralizing antibody serums and Y. Mahida for the Vero cells. We are also grateful to A. Olling and R. Gerhard for methodological advice on Western blots.

Author Contributions The study was conceived by N.M. and designed by S.K., S.C. and J.H.

Construction of mutants and in vitro characterisation was carried out by S.K. In vivo work was carried out by S.C., M.K. and A.C. Analysis of data was carried out by S.K. and M.K. with assistance from S.C. and J.H. The manuscript was written by S.K. and S.C. with critical input from all other authors. Funding for the study was sourced by N.M. and A.C.

Author Information Correspondence should be addressed to N.M. (nigel.minton@ nottingham.ac.uk) 
Figure 1. Characterization of $C$. difficile toxin mutants. a, The pathogenicity locus PaLoc of $C$. difficile 630 showing the intron insertion sites for the toxin mutants. b. Southern blot using an intron specific probe. The control plasmid (pMTL007C-E2) and the genomic DNA of the four strains was digested with EcoRV, which resulted in a band of ca. $9 \mathrm{~kb}$ for the plasmid, $10 \mathrm{~kb}$ for the $t c d B$ mutation (in $A^{+} B^{-}$and $A^{-} B^{-}$), just over $3 \mathrm{~kb}$ for the $t c d A$-single mutant $\left(A^{-} B^{+}\right)$and 1.8 and $1.2 \mathrm{~kb}$ for the $t c d A$-mutation in the double mutant, due to an additional EcoRV site in the catP gene. c, Western blot probing culture supernatants with anti-TcdA-antibody (tgcBIOMICS). d, Western blot probing culture supernatants with anti-TcdB-antibody (tgcBIOMICS).

Figure 2. In vitro cytotoxicity. $\mathbf{a}$ and $\mathbf{b}$, Supernatants of the parental strain $A^{+} B^{+}$and the three mutants $A^{-} B^{+}, A^{+} B^{-}$and $A^{-} B^{-}$were used in cell culture assays to measure cytotoxicity. HT29 cells (a) and Vero cells (b) were cultured to a flat monolayer before adding $C$. difficile supernatants in 4 -fold dilutions series. After a $24 \mathrm{~h}$ incubation toxin endpoint titres were determined. Data represent the mean \pm s.d.; $\mathbf{n}=3$. $\mathbf{c}$ and $\mathbf{d}$, Toxin neutralization assays. Appropriate dilutions of supernatants were pre-incubated with a suitable concentration of anti-TcdA or anti-TcdB serum for $1 \mathrm{~h}$ at $37^{\circ} \mathrm{C}$ and then added to HT29 cells (c) and Vero cells (d) which were evaluated after $24 \mathrm{~h}$. Scale bar represents $2 \mathrm{~mm}$.

Figure 3. Virulence of $\boldsymbol{C}$. difficile strains in hamsters. Groups of 8 hamsters were challenged with $C$. difficile $630 \Delta \operatorname{erm}\left(\mathrm{A}^{+} \mathrm{B}^{+}\right)$, or one of the toxin mutant strains, $A^{+} B^{-}, A^{-} B^{+}$or $A^{-} B^{-}$. a, Colonisation of Golden Syrian hamsters by each 
strain is presented as time from inoculation to colonisation in days. $n=8 . \mathbf{b}$, Time from colonisation to death. The duration of the experiment was set at 14 days. $\mathrm{n}=8$.

\section{Methods}

Strains and growth conditions. Strains used in this study were E. coli TOP10 (Invitrogen) as a cloning host, $E$. coli $\mathrm{CA} 434^{23}$ as a conjugal donor and $C$. difficile $630 \Delta \mathrm{erm}^{8}$ and mutants. All strains were stored at $-80^{\circ} \mathrm{C}$ stocks upon arrival and maintained as frozen stocks ever since. E. coli cultures were grown on Luria Bertani medium, aerobically, $37^{\circ} \mathrm{C}$ and shaking if liquid unless stated otherwise. C. difficile cultures were grown in $\mathrm{BHIS}^{24}$ or $\mathrm{TY}^{25}$, anaerobically, $37^{\circ} \mathrm{C}$ in an anaerobic workstation (Don Whitley, UK). Antibiotics were used at the following concentrations where appropriate: Chloramphenicol ( $25 \mu \mathrm{g} / \mathrm{ml}$ or $12.5 \mu \mathrm{g} / \mathrm{ml})$, thiamphenicol (15 $\mu \mathrm{g} / \mathrm{ml})$, spectinomycin $(250 \mu \mathrm{g} / \mathrm{ml}$ or $750 \mu \mathrm{g} / \mathrm{ml})$, erythromycin $(2.5 \mu \mathrm{g} / \mathrm{ml})$, Dcycloserine $(250 \mu \mathrm{g} / \mathrm{ml})$ and cefoxitin $(8 \mu \mathrm{g} / \mathrm{ml})$.

Molecular biology techniques. Qiagen mini prep kits were used to purify plasmids. Genomic DNA was obtained by phenol-chloroform extraction. Digests, PCRs and DNA purification were all done according to general protocols ${ }^{26}$. DNA sequencing was performed by Geneservice, UK.

Construction and characterization of mutants. The $C$. difficile single mutant strains $\mathrm{AB}^{-}$and $\mathrm{A}^{+} \mathrm{B}^{-}$were made using ClosTron technology as described previously ${ }^{5,6}$. The $\mathrm{A}^{-} \mathrm{B}^{-}$double mutant strain was made from the $\mathrm{A}^{+} \mathrm{B}^{-}$mutant using a cat $P$-based ClosTron using the 'pseudo-suicide' vector principle as described elsewhere ${ }^{25}$. The following 
retargeted plasmids pMTL007C-E2::Cdi- $t c d A-1584$ s, pMTL007C-E2::Cdi- $t c d B-1511 \mathrm{a}$ and for the double pMTL007S-C7::Cdi-tcdA-1584s were used. To verify the correct insertions, primers used for $t c d A$ were: Cdi-tcdA-F2 (5'-

TCAATTGACAGAACAAGAAATAAATAGTCTATGGAGC-3’) and EBS universal ${ }^{5}$, and Cdi-tcdA-R2 (5'-TACCCCATTGTCTTCAGAAAGAGATCCACC-3’') and ErmRAM-R (5'-ACGCGTGCGACTCATAGAATTATTTCCTCCCG-3’); and for $t c d B$ were: Cdi-tcdB-F1 (5'-TGATAGTATAATGGCTGAAGCTAATGCAGATAATGG3') and ErmRAM-R, and Cdi-tcdB-R1 (5'-

CTTGCATCGTCAAATGACCATAAGCTAGCC-3’) and EBS universal.

Southern blotting. Mutants were verified by Southern blot using an intron specific probe. $2 \mu \mathrm{g}$ genomic DNA were digested with EcoRV (NEB) overnight. The blot was carried out using a DIG high prime labelling and detection kit (Roche) according to the manufacturer's instructions.

Western blotting. Supernatants from $96 \mathrm{~h}$ cultures, grown anaerobically in TY, were concentrated 8-fold by chloroform-methanol-precipitation. Proteins were standardized and run on Tricine gels 10-20\% (Invitrogen) and transferred onto nitrocellulose membrane. The membranes were blocked with milk powder and then incubated with mouse monoclonal anti-TcdA antibody TTC 8 and mouse monoclonal anti-TcdB antibody $2 \mathrm{CV}$ (tgcBIOMICS) respectively, followed by protein A-HRP (Sigma). The ECL Western blot detection kit from Amersham was used according to the manufacturer's instructions.

Cell toxicity assays. The four strains were grown overnight in $5 \mathrm{ml} \mathrm{TY}$ under anaerobic conditions as previously described ${ }^{22}$, then the cell density was standardised, the cells centrifuged and supernatants filtered. Supernatants were diluted in a 4-fold series and 20 $\mu 1$ of dilutions were added onto monolayers of Vero and HT29 cells preincubated in 96 well plates for $48 \mathrm{~h}\left(\right.$ at $37^{\circ} \mathrm{C}, 5 \% \mathrm{CO}_{2}$ ). Cytotoxicity was recorded after $24 \mathrm{~h}$. For the 
neutralization assay appropriate dilutions of supernatants were pre-incubated with a suitable concentration of anti-TcdA or anti-TcdB serum (polyclonal, tgcBIOMICS) for $1 \mathrm{~h}$ at $37^{\circ} \mathrm{C}$. These were then added to Vero and HT29 cells which were evaluated after 24 h.

Vero and HT29 cells were grown in DMEM or McCoy's 5A, respectively, with 10\% v/v foetal calf serum and $1 \% \mathrm{v} / \mathrm{v}$ penicillin-streptomycin at $37^{\circ} \mathrm{C}, 5 \% \mathrm{CO}_{2}$ until confluent. Cells were detached using trypsin, and seeded into 96 well plates at a density of ca. $2 \times$ $10^{5}$ cells $/ \mathrm{ml}$. All assays were carried out in triplicate. GraphPad Prism was used for statistical analysis. Significant differences were assessed using one way ANOVA tests.

qRT-PCR. The qRT-PCR was carried out as described elsewhere ${ }^{4}$.

Hamster infection model. We used a block design with final group sizes of 8 animals. Female Golden Syrian hamsters (100 - 130 g) were housed singly in individually ventilated cages. Each hamster was dosed with clindamycin $(30 \mathrm{mg} / \mathrm{kg}) 5$ days prior to being infected orally with 100 spores each. Hamsters were monitored for signs of infection and sacrificed when the endpoint was met. The hamsters were handled individually in a microbiological safety cabinet. In line with UK Home Office requirements to reduce animal suffering, an alternative to death was used as the end point. Animals were monitored 3-4 times per day following infection and were assessed for several parameters including presence and severity of diarrhoea, weight-loss, level of activity, starey coat, sunken eyes, hunched posture and response to stimulus. A scoring system based on severity of changes observed (ranging from 0-3 for each parameter) was used to quantify changes in the condition of the animals which were euthanised when a pre-determined cumulative value was reached.

Faecal pellets were collected daily and plated to determine the presence of $C$. difficile. Caecum samples from each hamster were homogenized, plated and C. difficile counts 
obtained. PCR was performed to determine the genotype of each strain recovered from hamsters. Faecal and caecum samples were plated on Fructose agar (Clostridium difficile agar base, Oxoid) with cycloserine cefoxitin, taurocholate, tetracycline and amphotericin to select for Clostridium difficile. The following primer sets were used to authenticate the various strain genotypes: oligonucleotides 3800 and $10050^{8}$ to confirm the cells were derived from $C$. difficile 630 4 erm, oligonucleotide primers Cdi-tcdA-F2 (5'-TCAATTGACAGAACAAGAAATAAATAGTCTATGGAGC-3') and Cdi-tcdAR2 (5'-TACCCCATTGTCTTCAGAAAGAGATCCACC-3’) to distinguish between the toxin A insertional mutants and wildtype, and the primers Cdi-tcdB-F1 (5'TGATAGTATAATGGCTGAAGCTAATGCAGATAATGG-3') and Cdi-tcdB-R1 (5'CTTGCATCGTCAAATGACCATAAGCTAGCC-3') to distinguish between the toxin B insertional mutation and wildtype. A figure showing annealing of the primers and gel pictures can be found in supplementary information, which is linked to the online version of the paper at www.nature.com/nature.

23. Williams, D. R., Young, D. I. \& Young, M. Conjugative plasmid transfer from Escherichia coli to Clostridium acetobutylicum. J Gen Microbiol 136, 819-26 (1990).

24. Sorg, J. A. \& Sonenshein, A. L. Bile salts and glycine as cogerminants for Clostridium difficile spores. J Bacteriol 190, 2505-12 (2008).

25. Cartman, S. T. \& Minton, N. P. A mariner-based transposon system for in vivo random mutagenesis of Clostridium difficile. Appl Environ Microbiol 76, 1103-9 (2010).

26. Sambrook, J. \& Russell, D. W. Molecular Cloning - a Laboratory Manual (Cold Spring Habour, Laboratory Press, Cold Spring Habour, NY, 2001). 